\title{
Psychopharmakologieforschung in der Kinder- und Jugend- psychiatrie - Entwicklungen, Herausforderungen, Perspektiven
}

\author{
Psychopharmacology research in child and adolescent \\ psychiatry - Developments, challenges, perspectives
}

\author{
Ralf W. Dittmann¹ und Aribert Rothenberger ${ }^{2}$ \\ AG Klinische Psychopharmakologie des Kindes- und Jugendalters, Klinik für Psychiatrie und Psychotherapie des Kindes- und \\ Jugendalters, Zentralinstitut für Seelische Gesundheit, Mannheim/Medizinische Fakultät Mannheim, Ruprecht-Karls-Universität \\ Heidelberg, Deutschland \\ Klinik für Kinder- und Jugendpsychiatrie/Psychotherapie, Universitätsmedizin, Georg-August Universität Göttingen, Deutschland
}

\section{Einleitung}

Eine Behandlung mit Psychopharmaka gehört in der Kinder- und Jugendpsychiatrie (KJP) zu den Kernelementen des Behandlungsinstrumentariums für viele Störungsbilder. Angaben zu den Substanzen und ihrer Anwendung finden sich dementsprechend in den Leitlinien der Arbeitsgemeinschaft der Wissenschaftlichen Medizinischen Fachgesellschaften (AWMF, 2019). Allerdings ist die derzeitige Situation quantitativ und qualitativ unbefriedigend, sodass mehr und bessere Forschung wünschenswert ist.

In den letzten Jahrzehnten ist ein deutlicher Anstieg der Verordnungszahlen für diese Altersgruppe festzustellen (Rapoport, 2013; Steinhausen, 2015). Andererseits besteht nur für wenige psychotrope Substanzen eine entsprechende behördliche Zulassung (Kölch \& Plener, 2016; Persico et al., 2015), sodass Behandelnde häufig off label verordnen (müssen), mit Implikationen für z. B. Haftung, Kostenerstattung und Akzeptanz/Adhärenz (Gerlach \& Warnke, 2016).

Bei großen internationalen Unterschieden in Verordnungshäufigkeiten wird die Frage der Über-/Unterversorgung mit Psychopharmaka im Fachgebiet seit Längerem kontrovers diskutiert (Rapoport, 2013; Steinhausen, 2013; Taylor, 2013). Eine besser ausgestattete, zielgerichtete und innovative Klinische Psychopharmakologie des Kindesund Jugendalters (KPPKJ) könnte Lösungen für dieses Problem entwickeln.

\section{Forschungssituation in der KPPKJ}

Traditionell wird die präklinische und klinische Forschung und Entwicklung von Medikamenten weltweit primär von der forschenden pharmazeutischen Industrie vorgenommen, mit dem Ziel, behördliche Zulassungen, Vermarktung und finanzielle Erstattungen zu erreichen. Diese Prozesse unterliegen zwar staatlichen Rahmenbedingungen und Kontrollmaßnahmen, werden aber hinsichtlich Substanzauswahl und Indikationen weitgehend von den Herstellerfirmen bestimmt.

Seit den 1990er-Jahren wurden in den USA und Europa regulatorische Maßnahmen ergriffen, um die klinische Forschung und Entwicklung von Medikamenten für Kinder und Jugendliche (qualitativ hochwertig, ethisch) zu fördern, die Zahl der Zulassungen zu steigern, Off-LabelVerschreibungen zu verringern und damit die Qualität der medikamentösen Therapie zu verbessern. Pharmazeutische Unternehmen wurden verpflichtet, klinische Studien 
für diese Population durchzuführen (EU-Regulation 1901/2006; http://ec.europa.eu/enterprise/pharmaceuti cals/eudralex/vol1_en.htm), zuvor einen Pädiatrischen Prüfplan (PIP) bei den Zulassungsbehörden vorzulegen und abzustimmen. Patentrechtliche Anreize (Laufzeitverlängerung) und spezielle Zulassungen (Paediatric Use Market Authorization [PUMA]) wurden etabliert. Diese Neuregelungen gelten für von der Industrie gesponserte und öffentlich/universitär initiierte Entwicklungsprojekte. Obwohl eine Vielzahl von PIPs eingereicht/beurteilt wurde, sind direkte Auswirkungen dieses Maßnahmenkataloges (z.B. PUMA) bisher als sehr begrenzt für die KPPKJ anzusehen (Kölch \& Plener, 2016; Persico et al., 2015; Schmäl et al., 2014; Vitiello \& Davico, 2018).

In diesem Zeitraum wurden damit aber deutlich mehr Entwicklungsvorhaben der pharmazeutischen Industrie angestoßen, auch vermehrt finanzielle Förderungen für KPPKJ-Projekte (s.u.) von der EU (FP7 Förderprogramm) oder anderen Förderinstitutionen (z. B. Bundesinstitut für Arzneimittel und Medizinprodukte, Bundesministerium für Bildung und Forschung). Dadurch wurde die Zusammenarbeit zwischen KPPKJ-engagierten Zentren intensiviert, mit der Etablierung von inter-/nationalen Konsortien und Netzwerken (s.u.).

Öffentlich und von der Pharmaindustrie geförderte Projekte haben jedoch eine Reihe von Herausforderungen in der Realisierung, Etablierung und Durchführung von klinischen (Zulassungs-) Studien der KPPKJ gezeigt, die nicht immer zufriedenstellend gelöst werden konnten (siehe Beispiele in Tabelle 1):

In Deutschland ist darüber hinaus zu beobachten, dass sich bei inter-/nationalen klinischen Entwicklungsprozessen nur eine begrenzte Anzahl von Zentren zu Studienteilnahmen bereitfindet (Schmäl et al., 2014). Eine (Stiftungs-) Professur für KPPKJ wurde 2008, erstmals in Europa, am ZI Mannheim eingerichtet, aus der sich eine KPPKJ Arbeitsgruppe entwickelt hat (ZI).

Einzelne KPPKJ-Entwicklungsprojekte der internationalen Pharmaindustrie (z.B. zu Antidepressiva: Agomelatin, Atypika: Lurasidon) sind von zuständigen Ethikkommissionen der jeweiligen Prüfleiterinnen und -leiter in Deutschland in den letzten Jahren nicht genehmigt worden. Die Firmen verteilten daher für Deutschland vorgesehene Studienpopulationen auf andere außer-/europäische Länder. Üblichen Firmenaktivitäten für die Zulassung/ Vermarktung wurde demzufolge kaum Priorität zugemessen. Diese Entwicklung stellt einen erheblichen Verlust für den Forschungsstandort Deutschland dar, aber auch für die Vielfalt in der medikamentösen Versorgung unserer Patientenpopulation. Negative Implikationen für die Bereitschaft der Öffentlichkeit, von Patientinnen und Patienten sowie deren Eltern, an klinischen Studien in diesem Feld teilzunehmen, müssen angenomommen werden.
Ein ähnliches Reaktionsmuster zeigten Unternehmen, für deren - z.T. auch für die KJP bereits zugelassene - Substanzen, z.B. Lisdexamphetamin (LDX), im Rahmen des Arzneimittelneuordnungsgesetz(AMNOG)-Verfahrens vom Gemeinsamen Bundesausschuss (GBA) bzw. des Instituts für Qualität und Wirtschaftlichkeit im Gesundheitswe-

Tabelle 1. Herausforderungen.

- Von unabhängigen Verwaltungsinstitutionen und Ethikkomissionen national/international differente, z. T. widersprüchliche Anforderungen an einen Prüfplan

- Abstimmung von PIP in öffentlich-gefördertem Projekt mit Zulassungsbehörden (z. B. EMA) für eine PUMA (Risperidon, neue Indikation) zeitaufwendig und begrenzend für Studiendesign

- Öffentliche Förderung verlangt Kooperation mit kleinen/ mittleren Unternehmen (SMEs/CROs), Großteil der Fördersumme dafür gebunden

- Deren Leistungsfähigkeit/Zuverlässigkeit für ein Uni-Konsortium kaum zu beurteilen; Teilprojekte z. B. gescheitert, da eine Generika-Firma die Prüfmedikation (Risperidon, Plc) wegen Unzulänglichkeiten (in GMP) auf Behördenannordnung nicht ausliefern durfte; Ersatz nicht möglich (Glennon et al., 2014; https://cordis. europa.eu/project/rcn/95491 de.html). Forschende Pharmafirmen haben größere Flexibilität in Zeitplan und Finanzvolumen

- Rekrutierung von KPPKJ-Studienpatientinnen und - patienten oft schwierig (bei PIP, engen Ein-/Ausschlusskriterien); so nur eine sehr kleine Teilgruppe der Routine-Patientenpopulation eingeschlossen (z.B. Duloxetin; Bliznak, Berg, Häge \& Dittmann, 2013) bzw. Studienprojekt vorzeitig beendet (Johanniskraut; DEMIJO; Bundesministerium für Bildung und Forschung). Begrenzte Repräsentativität, damit Evidenz (auch für Leitlinien), ggf. eingeschränkte Übertragbarkeit auf klinische Versorgungssituation zu berücksichtigen

- Personalsituationen und Infrastrukturen (z. B. beklagt von der Deutschen Forschungsgesellschaft, Wissenschaftsrat, Forum Gesundheitsforschung, der Arbeitsgemeinschaft der Wissenschaftlichen Medizinischen Fachgesellschaften; Richter-Kuhlmann, 2019): u.a. wenige studienerfahrene Mitarbeiterinnen und Mitarbeiter zur Verfügung bzw. zu gewinnen

- Klinischen/universitären Konsortien fehlen oft wesentliche in der Industrie fest verankerte Kompetenzen, z. B. zu den Bereichen Zulassung/sbehörden, Ethikkomissionen, Vertragsrecht, Arzneimittelsicherheit und Reporting, Monitoring/Qualitätssicherung, Patientenversicherung, Prüfmedikation/Drug Supply, evtl. Studiendesign, Biometrie. Missverständnisse, Verzögerungen, Kosten für spezialisierte Firmen (CROs, KKS etc.) als Folge. Erfreulicherweise erste Kooperationen zwischen öffentlichen und privaten Organisationen (EC; EFPIA), wie IMI (Meulien, 2019)

Anmerkungen. PIP = Paediatric Investigation Plan; EMA = European Medicines Agency; PUMA = Paediatric Use Market Authorization; SME = Small/ Medium Enterprise; $\mathrm{CRO}=$ Contract Research Organisation; GMP = Good Manufacturing Practice; KPPKJ = Klinische Psychopharmakologie des Kindes- und Jugendalters; DEMIJO = Depressionsbehandlung bei Minderiährigen mit Johanniskraut; KKS = Koordinationszentrum für Klinische Studien; $\mathrm{EC}=$ European Commission; EFPIA = European Federation of Pharmaceutical Industries and Associations; IMI = The Innovative Medicines Initiative. 
sen kein Zusatznutzen festgestellt wurde. Unterschiedliche Anforderungen an Studiendesigns - im Zulassungsverfahren einerseits, im nachfolgenden Verfahren zur Nutzenbewertung andererseits - spielten eine zentrale Rolle; z.B. wurden Studiendesignaspekte und Outcomes/Ergebnisse aus Zulassungsstudien für die zeitlich nachgelagerte Nutzenbewertung nicht anerkannt. Umfangreiche den Zusatznutzen befürwortende Stellungnahmen aus KJP-Expertenkreisen vermochten im o.g. Fall (LDX) den GBA nicht zu überzeugen (vgl. Dittmann, 2016).

Diese Gesamtsituation wurde häufig begleitet von Medienberichten über „unsolide, gefährliche“ Studienvorhaben in der KJP oder zu vermuteten Interessenkonflikten bei Studienleitern/Ärzten. Dies kann erheblich zur Verunsicherung von Eltern/Patientinnen und Patienten beitragen, mit u.a. entsprechenden Auswirkungen auf die Repräsentativität der Studienteilnehmerinnen und -teilnehmer (s.o.).

\section{Neuere Entwicklungen}

Im letzten Jahrzehnt kam es zu intensiverer Unterstützung dieses Arbeitsbereiches durch öffentliche Forschungsförderungen, primär das EU-Forschungsprogramm FP7 (EUFP7, 2007) mit europäischen multinationalen Projekten, zu verschiedenen KPPKJ-Aspekten (siehe Tabelle 2):

Einige dieser Studienkomplexe sind noch nicht abgeschlossen. Bei anderen konnte leider ein größerer Teil der Untersuchungen aus diversen Gründen, auch Zeit- und Budgetbegrenzungen, nicht wie geplant umgesetzt werden, Umstände, welche vielfältige, z.T. neue Fragen zur Forschungsethik aufwerfen (Dittmann, 2018). Es sind jedoch - selbst nach Projektlaufzeitende - noch wesentliche Publikationen zu erwarten.

Weitere jüngere Initiativen - i.d.R. ohne Finanzierung durch Drittmittel - konzentrierten sich z. B. auf (Netzwerk)Meta-Analysen zur Medikamentenwirksamkeit (Cortese et al., 2017; Kanters et al., 2016), unzureichende Medikamenten-Adhärenz (Häge et al., 2018; Niemeyer et al., 2018), Netzwerkstrukturen (Persico et al., 2015)) sowie ethische Fragestellungen bei klinischer Anwendung und Forschung zu Psychopharmaka in der KJP (Dittmann, 2018; Gerlach \& Warnke, 2016; Rothenberger \& Rothenberger, 2012).

In diesem Heft werden drei ausgewählte Aspekte aktueller KPPKJ in Form von Artikeln aufgegriffen, nämlich Adhärenz (Mechler \& Häge, 2019), Langzeitmedikation bei der Aufmerksamkeitsdefizit-/Hyperaktivitätsstörung (ADHS; Taylor, 2019) und Belohnungsverarbeitung als Wirkmechanismus und transdiagnostisches Merkmal, hier am Beispiel der Depression im Jugendalter (Stringaris \& O'Callaghan, 2019). Die Artikel sind aus Vorträgen
Tabelle 2. Projekte und Themen, Beispiele.

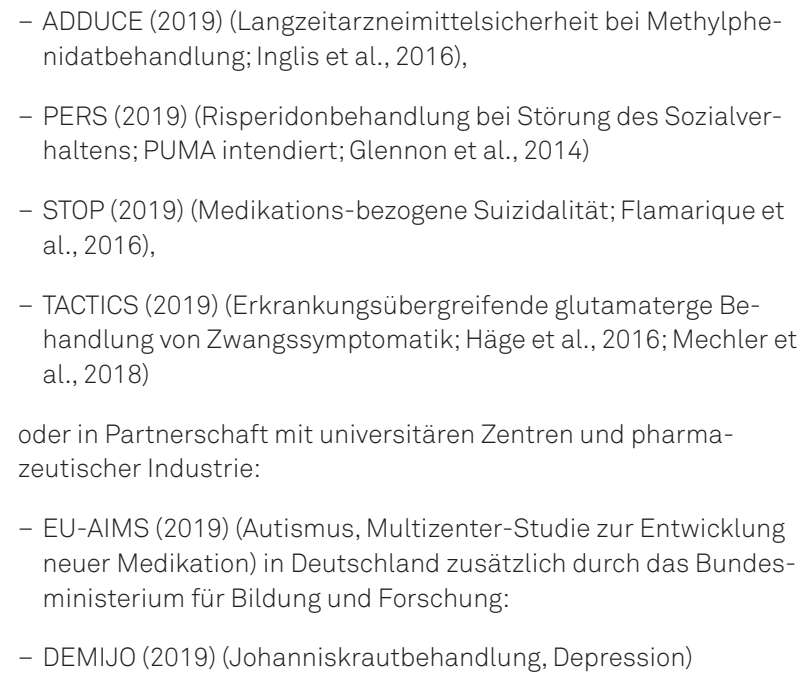

- EU-AIMS (2019) (Autismus, Multizenter-Studie zur Entwicklung neuer Medikation) in Deutschland zusätzlich durch das Bundesministerium für Bildung und Forschung:

- DEMIJO (2019) (Johanniskrautbehandlung, Depression)

oder das Bundesministerium für Gesundheit/Bundesinstitut für Arzneimittel und Medizinalprodukte:

- OLUNAR (2019) (Antidepressiva, Neuroleptika, Methylphenidat)

$-\operatorname{TDM}(2019)$

Anmerkungen. ADDUCE = Attention Deficit Hyperactivity Disorder Drugs Use Chronic Effects; PERS = The Pediatric European Risperidone Studies; PUMA = Paediatric Use Market Authorization; STOP = Suicidality: Treatment Occuring in Paediatrics; TACTICS = Translational Adolescent and Childhood Therapeutic Interventions in Compulsive Syndromes; EU-AIMS = European Autism Interventions - A Multicentre Study for Developing New Medications; DEMIJO = Depressionsbehandlung bei Minderjährigen mit Johanniskraut; OLUNAR = Arzneimittelsicherheit bei Kindern und Jugendlichen;TDM = Kompetenznetz „Therapeutisches Drug Monitoring Kinder-und Jugendpsychiatrie“.

im Rahmen des Abschiedssymposiums von Prof. Dr. Dr. R.W. Dittmann (Mannheim, Juni 2016) hervorgegangen.

Mechler und Häge (2019) machen in ihrer Übersicht deutlich, dass das Konzept der Adhärenz (am ehesten als „Therapietreue" zu verstehen) zwar schon vielfach betrachtet wurde, aber immer noch viele theoretische und praktische Unklarheiten bereithält. Wenn z. B. bei der Multimodal Treatment (MTA)-Studie zu ADHS eine vollständige Adhärenz von nur $53.5 \%$ berichtet wird, so ist hier nicht nur die Frage nach der praktischen Wertigkeit der Ergebnisse, sondern auch nach der Definition zu stellen. Letzteres wird offenbar sehr verschieden gehandhabt, je nachdem ob man das Zielkriterium eher kategorial (ja/nein) oder dimensional (als Prozentanteil) definiert. Von daher sind auch alle Kostenschätzungen wegen Nichtadhärenz sehr von der jeweiligen Definition abhängig. So kommt der Forschung zur validen, reliablen und einheitlichen Messung von Adhärenz eine wichtige Zukunftsaufgabe zu.

Dies ist gerade bei psychisch auffälligen Jugendlichen schwierig, da hier vielfältige Einflussfaktoren erfasst und kontrolliert werden müssen (z.B. mangelnde Wirksam- 
keit, Nebenwirkungen, Skepsis gegenüber Medikation, Autonomiebestrebung, Vergesslichkeit, Stigma, komplexe Dosierung, wechselnde Haltungen). Leider war die Suche nach Wegen, die Adhärenz von Jugendlichen zu verbessern, bisher wenig erfolgreich. Ob die noch laufende SEMA-Studie der Autoren hier einen Fortschritt bringen wird, bleibt zu hoffen, zumal das Thema für den klinischen Alltag, aber auch für den ökonomischen Einsatz von Therapiekosten eine große Bedeutung hat.

Es wirkt mit seinen vielfältigen Aspekten auch in den Bereich der Langzeitmedikation hinein. Taylor (2019) kommt in seinem „Perspektivartikel“, u. a. am Beispiel der MTA-Studie, darauf zurück und stellt dabei die vertrauensvolle Arzt-Patient-Familie-Beziehung heraus. Darüber hinaus versucht Taylor zu klären, welche Gründe es für die oft nachlassende Wirkung (und damit verbundene Dosiserhöhung) einer langzeitigen ADHS-Medikation mit Stimulanzien geben könnte.

Zum einen könnten im Laufe der Behandlung, die ja auf die Kernsymptome von ADHS gerichtet ist, emotionale oder zwanghafte Merkmale in den Vordergrund treten und somit bei fehlender Anpassung von Diagnose/Therapie die Adhärenz der Jugendlichen verschlechtern. Diese und andere Besonderheiten des ADHS-Verlaufs erschweren die Forschung mit praxisrelevanten randomisiert kontrollierten Studien (RCTs) und lenken den Blick auf kreative Designs von Beobachtungsstudien.

Zum anderen muss auch die Möglichkeit einer neuronalen Toleranzentwicklung gegenüber den Stimulanzien beachtet werden. Ob hierbei die Vermehrung der Dopaminrezeptoren/-transporter der einzige zugrunde liegende Mechanismus ist, bleibt weiter in der Diskussion. Glücklicherweise kann man offenbar durch „Medikamentenferien“ diesem Toleranzeffekt begegnen. Auch lässt sich daran denken, dass man im Rahmen einer individualisierten Therapie, und zwar in Abhängigkeit von der zu erwartenden Alltagsaufgabe, mit der oder dem Betroffenen gemeinsam eine variable Dosierung entwickelt. Allerdings kann dabei leicht der Gedanke an ein „Neuro-Enhancement" mit seinen ethischen Implikationen aufkommen.

Von dort ist es nicht weit zur Belohnungsverarbeitung, die nicht nur bei ADHS (Stichwort: Aversion gegenüber verzögerter Belohnung), sondern in etwas anderer Form (mangelnde/reduzierte Belohnungsverarbeitung) bei Jugendlichen mit einer Depression die Neurodynamik im Hintergrund darstellen kann. Stringaris und O'Callaghan (2019) bereiten in ihrer Übersichtsarbeit diesbezügliche Daten aus fMRT- und EEG/ERP-Untersuchungen auf. Die Autoren kommen zum Schluss, dass das gesamte Belohnungsnetzwerk gestört ist. Es fand sich eine geringere Hirnaktivität nach Belohnungsreizen im ventralen Striatum, der Insula, dem Thalamus und dem mittleren/hinteren Cingulum, wohingegen (wohl als Kompensationsver- such, um die Balance der fronto-striatalen Konnektivität zu bewahren) vor allem dorsolaterale und mediale Bereiche eine verstärkte Hirnaktivität zeigten. Ähnlich wie hier für den emotionalen Regelkreis gibt es auch neurobiologische Belege bei Tic-Störungen für den motorischen frontostriato-thalamischen Regelkreis. Ob sich die Hoffnung der beiden Autoren erfüllt, dass sich die untersuchten neurobiologischen Parameter (BOLD-Aktivität bzw. FeedbackRelated Negativity) einmal als verlässliche Biomarker zur klinischen Früherkennung von geminderter Belohnungsverarbeitung eignen werden, muss derzeit offenbleiben. Weitere, auch durch „pharmacological challenge“ geleitete Forschung auf dem Gebiet der "Striatalen Hypoaktivität“ und ihrer klinischen Bedeutung bei Depressivität ist auf jeden Fall zu empfehlen.

Insgesamt bleiben, über die drei genannten Ansätze hinaus, der KPPKJ reichlich Themen, sowohl auf der Ebene des Verhaltens als auch der Neurobiologie, um das Feld weiter zu entwickeln.

\section{Ausblick: Fragestellungen und Aufgaben für die Zukunft in der KPPKJ}

Vielfältige Überlegungen und Vorschläge sind in den letzten Jahren diskutiert worden, mit dem Ziel, wesentliche neue Erkenntnisse - jenseits der Ergebnisse aus Zulassungsstudien (RCTs) - zu gewinnen (u.a. in Holtmann \& Schimmelmann, 2016; Kölch \& Plener, 2016; Persico et al., 2015; Rapoport, 2013; Rothenberger, Gerlach \& Dittmann, 2010; Taylor, 2013; Vitiello, 2016; Vitiello \& Davico, 2018), die im Rahmen dieser Erörterung nur übersichtsartig genannt werden können (siehe Tabelle 3):

Wenn man berücksichtigt, dass ein hoher Anteil von KJP-Patientinnen und -Patienten nicht gut auf ein einzelnes Medikament anspricht, Komorbiditäten häufig sind und die Variabilität bei unerwünschten Ereignissen/Nebenwirkungen groß ist, wird - auch im Sinne einer „personalisierten Medizin“ - eine Palette möglichst zugelassener, alternativ $\mathrm{zu}$ verwendender Substanzen in jedem Indikationsbereich notwendig sein. Es erscheint auch zukünftig dringend notwendig, diesen klinisch wichtigen Sachverhalt den Entscheidungsträgern der beteiligten Institutionen intensiv zu vermitteln.

Die Forschung in der KPPKJ hat zwar im letzten Jahrzehnt deutliche Fortschritte gemacht, sie muss aber weiter vorangetrieben werden, denn die verbleibenden Defizite sind noch sehr groß. Das Vorgehen sollte sich an o.g. Themen orientieren, möglichst unter Beteiligung deutscher Studienzentren. Entsprechende Rahmenbedingungen mit 
Tabelle 3. Zukünftige Themen.

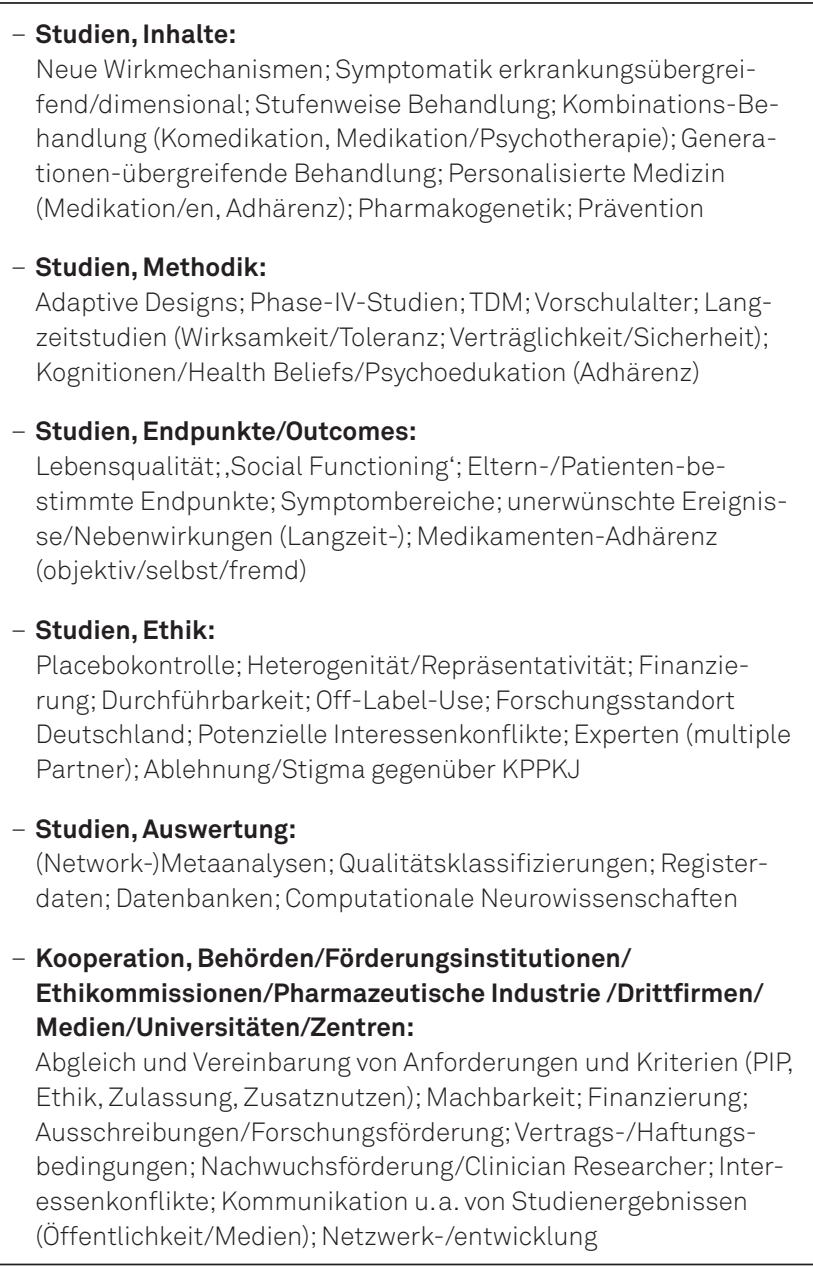

Anmerkungen. TDM = Kompetenznetz „Therapeutisches Drug Monitoring Kinder-und Jugendpsychiatrie“; KPPKJ = Klinische Psychopharmakologie des Kindes- und Jugendalters; PIP = Paediatric Investigation Plan.

nachfolgenden Zulassungen und Erstattungen durch das Gesundheitssystem sind zu fordern. Nur so kann die KPPKJ auch zukünftig dazu beitragen, eine bestmögliche medikamentöse Versorgung der betroffenen kinder- und jugendpsychiatrischen Patientinnen und Patienten sicherzustellen und weiterzuentwickeln.

\section{Literatur}

ADDUCE (2019). Attention Deficit Hyperactivity Disorder Drugs Use Chronic Effects. Retrieved from http://www.adhd-adduce.org/ AWMF (2019). Arbeitsgemeinschaft der Wissenschaftlichen Medizinischen Fachgesellschaften. Verfügbar unter: https://www. awmf.org/leitlinien/aktuelle-leitlinien.html

Bliznak, L., Berg, R., Häge, A. \& Dittmann, R.W. (2013). High rate of non-eligibility: Methodological factors impacting on recruit- ment for a multicentre, double-blind study of paediatric patients with major depressive disorder. Pharmacopsychiatry, 46(1), 23-28. doi:10.1055/s-0032-1314806

Cortese, S., Adamo, N., Mohr-Jensen, C., Hayes, A.J., Bhatti, S., Carucci, S. et al. (2017). Comparative efficacy and tolerability of pharmacological interventions for attention-deficit/hyperactivity disorder in children, adolescents and adults: Protocol for a systematic review and network meta-analysis. British Medical Journal Open, 7(1), e013967. doi:10.1136/bmjopen-2016-013967

DEMIJO (2019). Depressionsbehandlung bei Minderjährigen mit Johanniskraut-Studie. Verfügbar unter: http://www.gesund heitsforschung-bmbf.de/de/2852.php

Dittmann, R.W. (2016). Das Arzneimittelmarktneuordnungsgesetz aus kinder- und jugendpsychiatrischerPerspektive. Nervenarzt, 87(4), 367-375. doi:10.1007/s00115-016-0094-0

Dittmann, R.W. (2018). Ethical aspects of psychopharmacological research in children and adolescents. Paper presented at the 30th Conference 2018, FCAP, Royal Australian \& New Zealand College of Psychiatrists, Perth, Western Australia.

EU-AIMS (2019). European Autism Interventions - A Multicentre Study for Developing New Medications. Neuroscience \& Biobehavioral Reviews. Retrieved from http://www.eu-aims.eu/

EUFP7 (2007). EU 7th Framework Programme for Research. Retrieved from http://ec.europa.eu/research/fp7/index_en.cfm (2007\char212013)

Flamarique, I., Santosh, P., Zuddas, A., Arango, C., Purper-Ouakil, D., Hoekstra, P.J. et al. (2016). Development and psychometric properties of the Suicidality: Treatment Occurring in Paediatrics (STOP) Suicidality Assessment Scale (STOP-SAS) in children and adolescents. BioMed Central Pediatrics, 16(1), 213. doi:10.1186/s12887-016-0751-2

Gerlach, M. \& Warnke, A. (2016). Medikamentöse Behandlung in der Kinder- und Jugendpsychiatrie in Deutschland zwischen ethischen sowie sozial- und haftungsrechtlichen Konflikten. Zeitschrift für Kinder- und Jugendpsychiatrie und Psychotherapie, 44(4), 249-255.

Glennon, J., Purper-Ouakil, D., Bakker, M., Zuddas, A., Hoekstra, P., Schulze, U. et al. (2014). Paediatric European Risperidone Studies (PERS): Context, rationale, objectives, strategy, and challenges. European Child and Adolescent Psychiatry, 23(12), 1149-1160. doi:10.1007/s00787-013-0498-3

Häge, A., Banaschewski, T., Buitelaar, J. K., Dijkhuizen, R. M., Franke, B., Lythgoe, D.J. et al. (2016). Glutamatergic medication in the treatment of obsessive compulsive disorder (OCD) and autism spectrum disorder (ASD) - study protocol for a randomised controlled trial. Trials, 17(1), 141. doi:10.1186/s13063-016-1266-8

Häge, A., Weymann, L., Bliznak, L., Märker, V., Mechler, K. \& Dittmann, R.W. (2018). Non-adherence to psychotropic medication among adolescents - A systematic review of the literature. Zeitschrift für Kinder- und Jugendpsychiatrie und Psychotherapie, 46(1), 69-78. doi:10.1024/1422-4917/a000505

Holtmann, M. \& Schimmelmann, B. (2016). From psychopharmacological evidence to clinical practice: Unmet needs in child \& adolescent psychiatry - Reflections on Kölch \& Plener. Pharmacopsychiatry, 49(6), 227-228.

IMI (2019). The Innovative Medicines Initiative. Retrieved from https://www.imi.europa.eu/

Inglis, S.K., Carucci, S., Garas, P., Häge, A., Banaschewski, T., Buitelaar, J.K. et al. (2016). Prospective observational study protocol to investigate long-term adverse effects of methylphenidate in children and adolescents with ADHD: The Attention Deficit Hyperactivity Disorder Drugs Use Chronic Effects (ADDUCE) study. British Medical Journal Open, 6(4), e010433. doi:10.1136/ bmjopen-2015-010433

Kanters, S., Ford, N., Druyts, E., Thorlund, K., Mills, E.J. \& Bansback, N. (2016). Use of network meta-analysis in clinical guidelines. 
Bulletin World Health Organization, 94(10), 782-784. doi:10.2471/ BLT.16.174326

Kölch, M. \& Plener, P.L. (2016). Pharmaotherapy in psychiatric disorders of children: Current evidence and trends. Pharmacopsychiatry, 49(6), 219-225.

Mechler, K., Häge, A., Schweinfurth, N., Glennon, J.C., Dijkhuizen, R. M., Murphy, D. et al. (2018). Glutamatergic agents in the treatment of compulsivity and impulsivity in child and adolescent psychiatry: A systematic review of the literature. Zeitschrift für Kinder- und Jugendpsychiatrie und Psychotherapie, 46(3), 246263. doi:10.1024/1422-4917/a000546

Mechler, K. \& Häge, A. (2019). "Drugs Don't Work in Patients Who Don't Take Them". Zeitschrift für Kinder- und Jugendpsychiatrie und Psychotherapie, 47(6), 528-534.Meulien, P. (2019). Why we need public-private partnerships in drug R \& D. Retrieved from https://pharmaboardroom.com/articles/

Niemeyer, L., Schumm, L., Mechler, K., Jennen-Steinmetz, C., Dittmann, R. W. \& Häge, A. (2018). „When I stop my medication, everything goes wrong": Content analysis of interviews with adolescent patients treated with psychotropic medication. Journal of Child and Adolescent Psychopharmacology, 28(9), 655-662. doi:10.1089/cap.2018.0072

O'Callaghan, G. \& Stringaris, A. (2019). Reward Processing in Adolescent Depression Across Neuroimaging Modalities. Zeitschrift für Kinder- und Jugendpsychiatrie und Psychotherapie, 47(6), 535-541.

OLUNAR (2019). Arzneimittelsicherheit bei Kindern und Jugendlichen. Verfügbar unter: https://www.bips-institut.de/forschung/ projekte/einzelansicht.html?projid $=475$

PERS (2019). The Pediatric European Risperidone Studies. Retrieved from https://cordis.europa.eu/project/rcn/95491_de.html

Persico, A. M., Arango, C., Buitelaar, J.K., Correll, C.U., Glennon, J.C., Hoekstra, P.J. et al. (2015). Unmet needs in paediatric psychopharmacology: Present scenario and future perspectives. European Neuropsychopharmacology, 25(10), 1513-1531. doi:10.1016/j.euroneuro.2015.06.009

Rapoport, J.L. (2013). Pediatric psychopharmacology: too much or too little? World Psychiatry, 12(2), 118-123. doi:10.1002/wps.2028

Richter-Kuhlmann, E. (2019). Potenzial nicht ausgeschöpft - Klinische Studien. Deutsches Ärzteblatt, 116, 170-171.

Rothenberger, A., Gerlach, M. \& Dittmann, R.W. (2010). Psychotropic drugs in child and adolescent psychiatry - A new era on the horizon; Editorial. Current Pharmaceutical Design, 16(22), 2395-2397.

Rothenberger, A. \& Rothenberger, L.G. (2012). Updates on treatment of attention-deficit/hyperactivity disorder: Facts, comments, and ethical considerations. Current Treatment Options in Neurology, 14(6), 594-607. doi:10.1007/s11940-012-0197-2
Schmäl, C., Becker, K., Berg, R., Brünger, M., Lehmkuhl, G., Oehler K.U. et al. (2014). Pediatric psychopharmacological research in the post EU regulation 1901/2006 era. Zeitschrift für Kinderund Jugendpsychiatrie und Psychotherapie, 42(6), 441-449. doi:10.1024/1422-4917/a000322

Steinhausen, H.C. (2013). A European perspective on paedopsychiatric pharmacoepidemiology. World Psychiatry, 12(2), 131-132. doi:10.1002/wps.20034

Steinhausen, H.C. (2015). Recent international trends in psychotropic medication prescriptions for children and adolescents. European Child and Adolescent Psychiatry, 24(6), 635-640. doi:10.1007/s00787-014-0631-y

STOP (2019). Suicidality: Treatment Occuring in Paediatrics. Retrieved from http://www.stop-study.com/

TACTICS (2019). Translational Adolescent and Childhood Therapeutic Interventions in Compulsive Syndromes. Retrieved from http://www.tactics-project.eu/

Taylor, E. (2013). Pediatric psychopharmacology: Too much and too little. World Psychiatry, 12(2), 124-125. doi:10.1002/wps.20030

Taylor, E. (2019). ADHD Medication in the Longer Term. Zeitschrift für Kinder- und Jugendpsychiatrie und Psychotherapie, 47(6), 542-546.

TDM (2019). Kompetenznetz „Therapeutisches Drug Monitoring Kinder-und Jugendpsychiatrie“.Verfügbar unter: www.tdm-kjp.de/

Vitiello, B. (2016). Pediatric psychopharmacology. Pharmacopsychiatry, 49(6), 226-227. doi:10.1055/s-0042-112819

Vitiello, B. \& Davico, C. (2018). Twenty years of progress in paediatric psychopharmacology: accomplishments and unmet needs. Evidence Based Mental Health, 21(4), e10. doi:10.1136/ ebmental-2018-300040

ZI (2019). Zentralinstitut für Seelische Gesundheit Mannheim. Verfügbar unter: https://www.zi-mannheim.de/

\section{Prof. Dr. med. Dr. phil. Ralf W. Dittmann}

AG Klinische Psychopharmakologie

des Kindes- und Jugendalters

Klinik für Psychiatrie und Psychotherapie

des Kindes- und Jugendalters

Zentralinstitut für Seelische Gesundheit,

Mannheim

Medizinische Fakultät Mannheim,

Ruprecht-Karls-Universität Heidelberg

Deutschland

ralf.dittmann@zi-mannheim.de 